\title{
A Comment on Boyers et al.: "Eltrombopag for the Treatment of Chronic Immune or Idiopathic Thrombocytopenic Purpura: A NICE Single Technology Appraisal”
}

\author{
Rachel Allen • Andres Brainsky • Kelly Grotzinger • \\ Tito Roccia
}

Published online: 23 November 2012

(c) The Author(s) 2012. This article is published with open access at Springerlink.com

\section{Dear Editor}

In their publication of the National Institute for Health and Clinical Excellence (NICE) single technology appraisal of eltrombopag, Boyers et al. [1] report the results of the indirect treatment comparison (ITC) of eltrombopag and romiplostim, conducted by GlaxoSmithKline (GSK) [the manufacturer of eltrombopag] and made available to NICE as part of the single technology appraisal of eltrombopag. Subsequent to the appraisal process, GSK identified and corrected a methodological error in this analysis (available at http://www.gsk-clinicalstudyregister.com [study ID: 114014]). NICE were made aware of this error in November 2010. This correction was not published as part of the original assessment of eltrombopag and is not used in the Boyers et al. publication [1]. GSK would like to alert the readers of PharmacoEconomics to the corrected data and interpretation of this analysis. The nature of the error, the corrected results and the impact on the conclusions of the article are outlined below.

\section{Indirect Comparison Methodology}

The analysis is the indirect comparison of durable and overall platelet response between eltrombopag and romiplostim based on two trials with romiplostim and one with eltrombopag (RAISE; Randomized Placebo-Controlled Idiopathic Thrombocytopenic Purpura Study with Eltrombopag). The primary endpoint in the romiplostim

R. Allen $(\bowtie) \cdot$ A. Brainsky $\cdot$ K. Grotzinger $\cdot$ T. Roccia

GlaxoSmithKline Ltd, 1-3 Iron Bridge Road,

Stockley Park West, Uxbridge UB11 1BT, UK

e-mail: rachel.1.allen@gsk.com studies (the probability of achieving a durable response) [2] differs from the primary endpoint in RAISE: the odds of achieving a platelet count $\geq 50<400 \times 10^{9} / \mathrm{L}$ [3]. An indirect comparison of durable and overall response was performed on a post hoc analysis of RAISE and the romiplostim studies applying the Bucher method [4]. Although the eltrombopag and romiplostim studies were conducted in similar patient populations, there are important differences in study design and in the definitions of response that introduce uncertainty and bias into the comparison. This should be taken into account in any interpretation of the analysis.

The analyses originally provided to NICE and subsequently presented by Boyers et al. [1] used incorrect efficacy data extracted from a subset of patients in RAISE, which should have been taken from the intent-to-treat (ITT) population.

\section{Corrected Results}

The durable and overall response rates were recalculated using data from the ITT population.

The results of the indirect comparisons of platelet response (eltrombopag compared with romiplostim), updated using the ITT data, are presented in Tables 1 and 2. No evidence of a significant difference in the odds of achieving either a durable or an overall response was identified between the eltrombopag and romiplostim treatment groups in analyses of all patients, splenectomized patients and non-splenectomized patients.

\section{Interpretation}

There is inherent uncertainty in any indirect comparison and such analyses should be treated with caution. There are 
Table 1 Comparison of efficacy between eltrombopag and romiplostim: durable response

\begin{tabular}{|c|c|c|c|c|}
\hline Treatment & $\begin{array}{l}\text { OR vs. placebo } \\
(95 \% \mathrm{CI})\end{array}$ & Treatment & $\begin{array}{l}\text { OR vs. placebo } \\
(95 \% \mathrm{CI})\end{array}$ & $\begin{array}{l}\text { Indirect comparison eltrombopag vs. } \\
\text { romiplostim [OR }(95 \% \mathrm{CI})]\end{array}$ \\
\hline \multicolumn{5}{|l|}{ All subjects } \\
\hline Eltrombopag & $12.69(4.36,36.92)$ & Romiplostim & $40.02(5.26,304.70)$ & $0.32(0.03,3.14)$ \\
\hline \multicolumn{5}{|c|}{ Splenectomized subjects } \\
\hline Eltrombopag & $13.33(1.66,107.43)$ & Romiplostim & $26.77(1.52,472.41)$ & $0.50(0.01,17.32)$ \\
\hline \multicolumn{5}{|c|}{ Non-splenectomized subjects } \\
\hline Eltrombopag & $12.97(3.72,45.26)$ & Romiplostim & $31.25(3.81,256.24)$ & $0.41(0.04,4.80)$ \\
\hline
\end{tabular}

$O R$ odds ratio

Table 2 Comparison of efficacy between eltrombopag and romiplostim: overall response

\begin{tabular}{|c|c|c|c|c|}
\hline Treatment & $\begin{array}{l}\text { OR vs. placebo } \\
(95 \% \mathrm{CI})\end{array}$ & Treatment & $\begin{array}{l}\text { OR vs. placebo } \\
(95 \% \mathrm{CI})\end{array}$ & $\begin{array}{l}\text { Indirect comparison eltrombopag vs. } \\
\text { romiplostim [OR }(95 \% \mathrm{CI})]\end{array}$ \\
\hline \multicolumn{5}{|l|}{ All subjects } \\
\hline Eltrombopag & $13.96(6.12,31.86)$ & Romiplostim & $64.07(17.33,236.82)$ & $0.22(0.05,1.02)$ \\
\hline \multicolumn{5}{|c|}{ Splenectomized subjects } \\
\hline Eltrombopag & $14.25(2.98,68.02)$ & Romiplostim & $151.63(8.39,2741.84)$ & $0.09(0.00,2.52)$ \\
\hline \multicolumn{5}{|c|}{ Non-splenectomized subjects } \\
\hline Eltrombopag & $14.83(5.53,39.76)$ & Romiplostim & $43.20(9.27,201.33)$ & $0.34(0.06,2.14)$ \\
\hline
\end{tabular}

$O R$ odds ratio

several additional factors that increase the uncertainty of the analysis in this particular case:

- The main outcome of the comparison, durable response, was the primary endpoint of the romiplostim studies and a post hoc analysis for eltrombopag; this means that whilst the romiplostim studies were specifically planned to address the aforementioned outcome, RAISE was not.

- One of the outcomes presented by Boyers et al. [1] is overall response, which is the sum of 'durable response' and 'transient response'. Durable response was similarly defined in the romiplostim trials and the eltrombopag post hoc analysis: a response in at least six of the last eight visits of the treatment period. However, the definition of 'transient response' was different: a transient response in the romiplostim analysis required a response at any four, weekly visits during the study, whereas the eltrombopag analysis required four consecutive weekly visits. In a disease where platelet counts fluctuate, four consecutive responses are more difficult to achieve and this is likely to have biased the ITC against eltrombopag.

- Whilst the romiplostim study did not allow tapering or interruptions of concomitant immune thrombocytopenic purpura (ITP) medications during the last 12 weeks of study, in RAISE physicians were encouraged to reduce concomitant ITP medications once a stable dose of eltrombopag was achieved. This was more likely to occur towards the end of the trial, when durable response was assessed in the post hoc analysis. Platelet count fluctuations are expected as a result of tapering ITP medications, and this most likely negatively impacted the response estimates for eltrombopag.

- The number of durable and overall responders in the placebo arm of the romiplostim study was very low. As such, any indirect comparison will be very sensitive to small changes in this event rate.

\section{Conclusion}

The corrected ITC demonstrates that there is no evidence of a statistically significant difference in durable or overall response between eltrombopag and romiplostim for all patients, splenectomized patients or non-splenectomized patients. Results should be interpreted in the context of the significant uncertainty associated with this comparison, in an orphan disease area, where there is a paucity of robust evidence.

Acknowledgments All authors are employees of GlaxoSmithKline, the manufacturer of eltrombopag. 
Open Access This article is distributed under the terms of the Creative Commons Attribution Noncommercial License which permits any noncommercial use, distribution, and reproduction in any medium, provided the original author(s) and the source are credited.

\section{References}

1. Boyers D, Jia X, Jenkinson D, Mowatt G. Eltrombopag for the treatment of chronic immune or idiopathic thrombocytopenic purpura: a NICE single technology appraisal. Pharmacoeconomics. 2012;30(6):483-95.
2. Kuter DJ, Bussel JB, Lyons RM, Pullarkat V, Gernsheimer TB, Senecal FM, et al. Efficacy of romiplostim in patients with chronic immune thrombocytopenic purpura: a double-blind randomised controlled trial. Lancet. 2008;371(9610):395-403.

3. Cheng G, Saleh MN, Marcher C, Vasey S, Mayer B, Aivado M, et al. Eltrombopag for management of chronic immune thrombocytopenia (RAISE): a 6-month, randomised, phase 3 study. Lancet. 2011;377:393-402.

4. Bucher HC, Guyatt GH, Griffith LE, Walter SD. The results of direct and indirect treatment comparisons in meta-analysis of randomized controlled trials. J Clin Epidemiol. 1997;50(6): 683-91. 